\title{
Effects of Low-Intensity Pulsed Ultrasound on Pain and Functional Disability in Patients With Early- Stage Lumbar Spondylolysis: A Randomized Controlled Trial
}

\section{Fahad Tanveer}

University of Lahore

Syed Asadullah Arslan ( $\sim$ asadshahgilani@gmail.com )

University of Lahore

Haider Darain

Khyber Medical University

Ashfaq Ahmad

University of Lahore

Syed Amir Gilani

University of Lahore

Asif Hanif

University of Lahore

\section{Research Article}

Keywords: Functional disability, Lumbar vertebra, Low-intensity pulsed ultrasound, Pain, Spondylolysis

Posted Date: June 16th, 2021

DOl: https://doi.org/10.21203/rs.3.rs-598951/v1

License: (c) (i) This work is licensed under a Creative Commons Attribution 4.0 International License.

Read Full License

Version of Record: A version of this preprint was published at Journal of Bodywork and Movement Therapies on February 1st, 2022. See the published version at https://doi.org/10.1016/j.jbmt.2022.02.025. 


\section{Research Article}

Effects of low-intensity pulsed ultrasound on pain and functional disability in patients with early-stage lumbar spondylolysis: A randomized controlled trial

Fahad Tanveer, PT, DPT ${ }^{1}$

Syed Asadullah Arslan, PT, DPT, PhD ${ }^{1}$

Haider Darain, PT, DPT, PhD ${ }^{2}$

Ashfaq Ahmad, PT, DPT, PhD ${ }^{1}$

Syed Amir Gilani, $\mathrm{PhD}^{3}$

Asif Hanif, $\mathrm{PhD}^{4}$

${ }^{1}$ University Institute of Physical Therapy, University of Lahore, Lahore, Pakistan.

${ }^{2}$ Institute of Physical Medicine \& Rehabilitation, Khyber Medical University, Peshawar, Pakistan.

${ }^{3}$ Faculty of Allied Health Sciences, University of Lahore, Lahore, Pakistan.

${ }^{4}$ University Institute of Public Health, University of Lahore, Lahore, Pakistan.

Address correspondence to: Syed Asadullah Arslan, University Institute of Physical Therapy, University of Lahore, Defense Road, Lahore, Pakistan Postal Code: 54770, Phone number: +923321483575. E-mail: asadshahgilani@gmail.com 


\section{ABSTRACT}

Background: Low Intensity Pulsed Ultrasound (LIPUS) is beneficial in accelerating fracture recovery, enhancing their capacity to execute tasks of daily life and, as a result, their autonomy.

Methods: Thirty-four (29 males and 5 females) pre-diagnosed patients referred by an orthopaedic surgeon exhibiting symptomatic low back pain for at least four months were recruited and randomly divided into LIPUS group and Routine Physical Therapy (RPT) group. The lottery method was used to randomly assign patients into two groups. Numeric Pain Rating Scale (NPRS) was utilized for the measurement of pain and Oswestry Disability Index (ODI) for functional disability. Patients were assessed at baseline, at the end of $12^{\text {th }}$ and $20^{\text {th }}$ week. Interventions were applied by the physical therapist having more than eight years of clinical experience for 10 weeks on alternate days.

Results: LIPUS group reported greater reduction in pain with mean change of 3.18 points (95\% CI: $2.2,4.2 ; \mathrm{p}<0.001)$ at $12^{\text {th }}$ week, 6.18 points $(95 \%$ CI: $5.5,6.8 ; p<0.001)$ at $20^{\text {th }}$ week follow-up and functional disability with mean change of 28.24 points (95\% CI: 23.7 , $32.8 ; \mathrm{p}<0.001)$ at $12^{\text {th }}$ week and 39.47 points $(95 \%$ CI: $31.8,47.1 ; \mathrm{p}<0.001)$ at $20^{\text {th }}$ week follow-up compared with the RPT group.

Conclusion: Low-intensity pulsed ultrasound has significantly reduced pain and functional disability in patients with early-stage lumbar spondylolysis and could be preferred as a safe non-invasive treatment method for early bone healing.

Trial registration: WHO-Iranian registry of clinical trials (IRCT20200206046396N1, Dated: 02/05/2020).

Key Words: Functional disability, Lumbar vertebra, Low-intensity pulsed ultrasound, Pain, Spondylolysis

\section{BACKGROUND}


Spondylolysis, a stress fracture of the pars interarticularis of the vertebral arch can affect either the left or right side of the bone. The L5 bone is the site of the vast majority of these injuries, with the L4 bone being the second most likely to be affected. The acuity of the fracture line or bony defect can be divided into 3 stages: early, progressive, and terminal. A hairline fracture can be seen in the early stages. The fracture may have advanced to a broader degree in the progressive stage and the fracture in the terminal stage indicates nonunion with no chance of healing [1]. Prevalence of spondylolysis in general population, varies from around 6\% to $11.5 \%$ [2] and in professional athletes, the prevalence ranges from $7 \%$ to $8 \%$. Nearly half of all low back pain cases in young athletes have been attributed to spondylolysis [3].

Low back pain is aggravated by excessive standing and hyperextension. In nearly $80 \%$ of patients, bilateral hamstring tightness, posterior buttock pain spreading to the knees while standing or walking, and potential neurological abnormalities along the L5 or S1 dermatomes that indicate nerve root impingement are common lower extremity findings [4]. Repetitive microtrauma contributing to spondylolysis has been attributed to lumbar hyperextension along with rotation and loading in dancers, gymnasts, figure skaters, weight lifters, and football players [5]. The easiest way to diagnose and plan therapy for spondylolysis is to take a thorough medical history and physical examination [6]. Location, intensity, duration, quality, and exacerbating or alleviating variables can also be included in the pain definition. Precipitating recreation or workplace hazards are two other areas to study [7].

Radiography is used to confirm the clinical diagnosis. Overall, with a sensitivity of 0.82 and specificity of 0.96, [5] Oblique radiographs, [8] which show an appearance of a Scottie dog with a collar, can be used for suspected yet poorly visualized pars defects [9]

LIPUS uses frequencies ranging from 0.75 to $1.5 \mathrm{MHz}$ with intensities of less than $100 \mathrm{~mW} / \mathrm{cm} 2$ (usually about $30 \mathrm{~mW} / \mathrm{cm} 2$ ) and is pulsed at a ratio of $1: 4$. The transducer head is strapped or otherwise fixed in place and remains stationary for 20 minutes of therapy. For effective sound transmission to deeper tissues, US medium is used once again. In the United States, the FDA approved this modality in 1994 for the treatment of rapid healing of fresh fractures, and again in 2000 for the treatment of confirmed non-union fractures. The findings 
submitted to the FDA showed that LIPUS improved angiogenesis, chondrogenesis, and osteogenesis at all three stages of fracture recovery, including inflammatory, reparative, and remodelling. LIPUS causes micro-motion and mechanical stimulus, and thus follows Wolff's Law, which says that bone remodels in response to mechanical stresses. [10]

LIPUS can be effective in early bone healing and its effects should be explored by doing more researches. As far as researcher's knowledge, no randomized controlled trial has been conducted to determine the effects of LIPUS; therefore, the hypothesis of present study was to determine the difference between the effects of routine physical therapy and routine physical therapy along with LIPUS on pain and functional disability in patients with early stage lumbar spondylolysis.

\section{METHODS}

\section{Study design and procedure}

From $05 / 06 / 2020$ to $05 / 06 / 2021$, this randomized controlled trial was conducted as part of a $\mathrm{PhD}$ Physical Therapy project at the Department of Physiotherapy, University of Lahore Teaching Hospital, Lahore, Pakistan, using a non-probability purposive sampling technique. After approval from the Institutional Review Board (IRB-UOL-FAHS/690/2020, Dated: 22/01/2020) of the University of Lahore, this study was prospectively registered in the WHO-Iranian registry of clinical trials (IRCT20200206046396N1, Dated: 02/05/2020). Sample size was determined by using WHO sample size software version 2.0. It was calculated as 28 , by adding $20 \%$ drop out it was 17 in each group. Subjects were divided randomly into two equal groups (LIPUS group and RPT group). Parallel assignment was done. Lottery method was used to randomly assign patients into two groups. NPRS was utilized for the measurement of pain and ODI for functional disability. LIPUS group was treated by low intensity pulsed ultrasound along with routine physical therapy whereas RPT group by routine physical therapy alone.

\section{Participants}

Thirty-four (29 males and 5 females) pre-diagnosed patients referred by an orthopaedic surgeon exhibiting symptomatic low back pain for at least four months and were between 
the ages of twenty and forty were recruited after they signed a written informed consent form for this research. Patients with the history of neurological or autonomic deficits, other fracture or bony abnormalities, rheumatic disease, other spinal problems, post-menopausal female, osteoporosis and osteopenia were excluded from the study. It was a singled blinded study in which the assessor was kept blinded.

\section{Intervention}

\section{Control group (RPT)}

Trans Electrical Nerve Stimulation (TENS) was applied for three days a week for 20 minutes per session. The active electrode was positioned in the center of the painful back area, while the second electrode was positioned on the lateral aspect of the thigh. The output frequency was set to 4 to $8 \mathrm{~Hz}$, and the current intensity was increased until the patient reports discomfort. The severity is then lowered to a degree that the patient reported he could tolerate. [11].

Hamstrings were stretched for 15 seconds for three times a day which is helpful for improving spino-pelvic rhythm (lumbar motion/pelvic motion) which is achieved by the flexibility of these muscles, thus decreases tension to the lumbar region [12]. Strengthening of abdominal muscles (transversus abdominis and internal oblique) were performed in 3 sets of 10 repetitions on alternate days which has been shown to reduce pain and functional disability in spondylolysis patients. Thus, provides "stability" to the lumbar spine, maintaining a solid foundation for individuals to incorporate them into their functional activity patterns [13].

\section{Intervention group (RPT+LIPUS)}

The following parameters were used in the LIPUS device (Accusonic lipus; Metron Medical Australia, Ltd, Victoria, Australia): 1.1-MHz oscillation frequency, 1-kHz pulsed frequency, $100-\mathrm{mW} / \mathrm{cm} 2$ spatial intensity, $2 \mathrm{~ms}$ pulse duration, $100 \mathrm{~Hz}$ pulse repetition rate, $20 \%$ pulse duty cycle, and 20-minute duration on alternate days. Subjects were asked to lie down on a couch in a prone position; a thin $(1 \mathrm{~mm})$ film of aqua-sonic gel was used as a coupling medium, and a fixed treatment head was positioned over the injured side just lateral to the spine of the lumbar vertebra with the aid of a Velcro strap [10]. 


\section{Outcome and measurements}

NPRS is a 11-point scale for self-report of pain. The respondent selects a whole number (integers $0-10$ ) that best reflects the intensity (or other quality if requested of his/her pain whereas ODI is an extremely important tool that researchers and disability evaluators use to measure a patient's permanent functional disability scored between $0-100 \%$. Thus, higher the score more will be the disability. The subjects were diagnosed and assessed by the same orthopedic surgeon. The same assessor provided his expertise at baseline, at the end of 12th and 20th week. Interventions were applied by the physical therapist having more than eight years of clinical experience for 10 weeks on alternate days.

\section{Statistics}

Normality of the data was tested by using Shapiro-Wilk test. Statistically, significant value was agreed at the level of 5\%. Descriptive statistics of age, height, weight and BMI were calculated. A repeated measure ANOVA with sphericity assumed for the LIPUS group and with a Greenhouse-Geisser correction for RPT group between assessments points (baseline, 12th and 20th week follow-up). Pairwise comparisons using Bonferroni correction and independent t-test for between group comparisons (baseline, 12th and 20th week follow-up).

\section{RESULTS}

Flow chart (Fig. 1)

\section{Baseline characteristics of demographic variables}

LIPUS and RPT groups were not significantly different for demographic variables at baseline. Baseline characteristics of the patients are presented in (Table 1).

Within and between group comparison for pain and functional disability at $12^{\text {th }}$ week $\& 20^{\text {th }}$ week follow-up from baseline

A repeated measure ANOVA with sphericity assumed for the LIPUS group and with a Greenhouse-Geisser correction for RPT group determined that the mean NPRS and ODI 
scores for both groups were found statistically significant between assessments points (baseline, $12^{\text {th }}$ week and $20^{\text {th }}$ week follow-up) $\left(F_{2,32}=118.90 ; p<0.001\right.$ for the LIPUS group

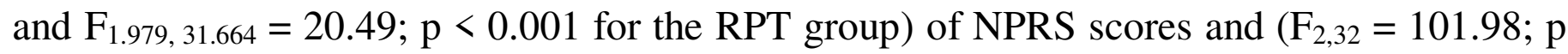
$<0.001$ for the LIPUS group and $\mathrm{F}_{1.152,18.436}=4.49 ; \mathrm{p}=0.043$ for the RPT group) of ODI scores respectively.

Pairwise comparisons using Bonferroni correction demonstrated that LIPUS group reported greater reduction in pain with mean change of 3.18 points (95\% CI: 2.2, 4.2; p < $0.001)$ at 12 th week, 6.18 points $(95 \%$ CI: 5.5, 6.8; p < 0.001) at 20th week follow-up and functional disability with mean change of 28.24 points $(95 \%$ CI: $23.7,32.8$; p < 0.001$)$ at 12th week and 39.47 points (95\% CI: 31.8, 47.1; p < 0.001) at 20th week follow-up compared with the RPT group (Table 2).

\section{DISCUSSION}

This study showed that the LIPUS has significantly reduced pain and functional disability in patients with early-stage lumbar spondylolysis. LIPUS group improved significantly in terms of pain and functional disability compared to RPT group. Therefore, the use of this treatment has a positive effect on patients with lumbar spondylolysis and may be used alone or in combination with other conventional techniques. Very limited researches were available showing the effects of LIPUS on spinal region which had conflicting results. Ebadi et al investigated the effects of LIPUS on chronic non-specific LBP and found low quality evidence of LIPUS on pain relief (MD [95 percent CI] -2.16 [-4.66 to 0.34]; zero to 50-point scale) and functional disability reduction (MD [95 percent $\mathrm{CI}]-2.16$ [-4.66 to 0.34$]$; zero to 50-point scale) (MD [95 percent CI] -0.41 [-3.14 to 2.32]; per cent) [14]. A similar study by Rubira et al compared the effects of LLLT and LIPUS on chronic non-specific low back pain and demonstrated a reduction in pain $(\mathrm{p}<0.001)$, with the LLLT (low-level lased therapy) group showing the greater relative increase (91.2 percent). Meanwhile, the RPT group showed a $-5.8 \%$ worsening of functional disability $(\mathrm{p}<0.001)$, but the LIPUS group showed the greatest relative improvement $(\mathrm{p}<0.001)(83.3$ percent) [15]. 
Different researches have also been conducted on other body regions to know its effects. A study by Zhou et al systemically reviewed the effect of LIPUS on pain and functional disability in knee osteoarthritis (KOA) patients. The visual analogue scale (VAS) and Western Ontario and McMaster Universities Osteoarthritis Index (WOMAC) scores were compared between the RPT and LIPUS groups using meta-analysis. In comparison to the RPT group, the LIPUS group found a decline in pain severity with mild heterogeneity ($0.79,95$ percent $\mathrm{CI},-1.57$ to $0.00 ; \mathrm{I} 2=65$ percent, $\mathrm{P}=0.04$ ) on the VAS and an increase in knee function on the WOMAC $(-5.30,95$ percent $\mathrm{CI},-2.88$ to $-7.71 ; \mathrm{I} 2=44$ percent, $\mathrm{P}=$ 0.17) [16]. Other studies by Jia et al and Raju et al determined the effect of LIPUS on knee osteoarthritis (KOA), and found major differences in VAS and WOMAC after 10 days of treatment, as well as improvements in VAS at 4 and 12 weeks. The mean age of patients in LIPUS and RPT group was $57.08 \pm 7.40$ and $58.04 \pm 9.93$ years respectively whereas in our study it was $30.47 \pm 5.01$ and 30.59 \pm 7.32 years respectively [17-18]. Munajat et al evaluated the effects of LIPUS in patients with Total Knee Arthoplasty and found significant $(\mathrm{P}<$ $0.01)$ reduction in pain and functional disability [19].

Pain and functional disability has been identified as the most disabling symptom in patients with lumbar spondylolysis. To address this problem a great deal of attention has been paid to specific therapies and supporting ancillary studies. Our results showed better effects on pain and functional disability in LIPUS group with a percentage change of $81 \%$ and $65 \%$ compared with $37 \%$ and $25 \%$ in RPT group. A study by D'vaz et al investigated the impact of LIPUS on chronic lateral epicondylitis and found that the LIPUS group had a 64 percent decrease in elbow pain from baseline compared to 57 percent in the RPT group (difference of 7\%; 95 percent confidence interval 20 to 35 percent). This was, however, not statistically significant $(\mathrm{P}=0.60)$ which might be due to lack of blinding and different predisposing factors effecting on elbow region [20]. Tehranchi et al determined the effect of LIPUS following mandibular surgery and discovered that the reduction in pain reported during the first three weeks after surgery was slightly different between groups $(\mathrm{P}<0.01)$ [21]. The study by Warden et al investigated the effectiveness of LIPUS in the reduction of pain symptoms and found no differences in VAS-U $(-0.2 \mathrm{~cm}$; 95 percent $\mathrm{CI},-1.5,1.1 \mathrm{~cm})(\mathrm{P}$ 
$=0.74)$ or VAS-W $(-0.5 \mathrm{~cm}$; 95 percent $\mathrm{CI},-2.1,1.1 \mathrm{~cm})(\mathrm{P}=0.57)$ were observed between the active- and inactive-LIPUS groups [22].

\section{CLINICAL IMPLICATIONS}

The findings of this study have several clinical implications for patients with early-stage lumbar spondylolysis as it is a non-invasive modality which can reduce pain and functional disability without disturbing the daily routine of the patients. Therefore, this approved to be an effective modality to be used by physiotherapists for the treatment of lumbar spondylolysis.

\section{STRENGTHS AND LIMITATIONS}

Strength of this study is our sample. We recruited from a variety of surgeons making our results generalizable. There are several limitations to this study. The first limitation was staging. We selected subjects with early-stage lumbar spondylolysis. The second limitation was the age of the subjects. The subjects who participated in this study were young. Therefore, the result of this study cannot be generalized to the entire population.

\section{CONCLUSION}

Low-intensity pulsed ultrasound has significantly reduced pain and functional disability in patients with early-stage lumbar spondylolysis and could be preferred as a safe non-invasive treatment method for early bone healing.

\section{Abbreviations}

LIPUS: Low-intensity pulsed ultrasound; NPRS: Numeric pain rating scale; ODI: Oswestry disability index; TENS: Trans electrical nerve stimulation (TENS); WOMAC: Western Ontario and Macmaster universities osteoarthritis index; VAS: visual analogue scale; KOA: knee osteoarthritis

\section{DECLARATIONS}


This study was conducted as part of a PhD Physical Therapy project after the approval from the Institutional Review Board (IRB-UOL-FAHS/690/2020, Dated: 22/01/2020) of the University of Lahore, prospectively registered in the WHO-Iranian registry of clinical trials (IRCT20200206046396N1, Dated: 02/05/2020). Subjects were recruited after their written informed consent for this study. All methods were performed in accordance with the relevant guidelines and regulations.

\section{Consent for publication}

Not applicable.

\section{Availability of data and materials}

The datasets generated and/or analyzed during the current study are not publicly available due to limitations of ethical approval involving the patient data and anonymity but are available from the corresponding author on reasonable request.

\section{Competing interests}

The authors declare that there is no conflict of interest.

\section{Funding}

None

\section{Authors' contributions}

FT, SAA, HD and AA contributed substantially to the conception and design of the work. SAG, AH was responsible for the acquisition of the data. AH was responsible for the analysis of the data. HD and $\mathrm{AH}$ made substantial contributions to the interpretation of the data. FT and SAA drafted the work and substantively revised it. The authors have read and approved the submitted version of the manuscript.

\section{Acknowledgements}


The authors thank Prof Imran and the University of Lahore Teaching Hospital for their assistance with patient recruitment, scheduling, and data management. Thank you to Prof Sohail for all his work on this study. Thank you to the University of Lahore Physical Therapy Clinic, Asim Arif, PT, DPT, and our other undergraduate and graduate lab assistants.

\section{Authors' information}

${ }^{1}$ University Institute of Physical Therapy, University of Lahore, Lahore, Pakistan.

${ }^{2}$ Institute of Physical Medicine \& Rehabilitation, Khyber Medical University, Peshawar, Pakistan.

${ }^{3}$ Faculty of Allied Health Sciences, University of Lahore, Lahore, Pakistan.

${ }^{4}$ University Institute of Public Health, University of Lahore, Lahore, Pakistan.

\section{REFERENCES}

1. Sakai T, Manabe H, Goto T. Extraosseous Signal Changes on Magnetic Resonance Imaging in Pediatric Patients with Early-Stage Lumbar Spondylolysis. J Med Invest. 2021; 68: 136-39. https://doi.org/10.2152/jmi.68.136

2. Urrutia J, Besa P, Lobos D. Lumbar paraspinal muscle fat infiltration is independently associated with sex, age, and inter-vertebral disc degeneration in symptomatic patients. Skeletal Radio. 2018; 47(7):955-61. https://doi.org/10.1007/s00256-018$2880-1$

3. Arnold A, Thigpen CA, Beattie PF. Overuse physeal injuries in youth athletes: risk factors, prevention, and treatment strategies. Sports Health. 2017; 9(2):139-47. https://doi.org/10.1177/1941738117690847

4. Ekin EE, Altunrende ME. Pedicle Stress Injury in Children and Adolescents with Low $\begin{array}{llll}\text { Back } & \text { Pain. } & \text { Spine. }\end{array}$ https://doi.org/10.1097/BRS.0000000000003046

5. Tawfik S, Phan K, Mobbs RJ. The incidence of pars interarticularis defects in athletes. Global Spine J. 2020; 10(1):89-101. https://doi.org/10.1177/2192568218823695 
6. Wang YX, Kaplar Z, Deng M. Lumbar degenerative spondylolisthesis epidemiology: a systematic review with a focus on gender-specific and age-specific prevalence. $\mathbf{J}$ Ortho Trans. 2017; 11:39-52. https://doi.org/10.1016/j.jot.2016.11.001

7. Raudenbush BL, Chambers RC, Silverstein MP. Indirect pars repair for pediatric isthmic spondylolysis: a case series. J Spine Surg. 2017; 3(3):387-390. https://doi.org/10.21037/jss.2017.08.08

8. Metzger R, Chaney S. Spondylolysis and spondylolisthesis: What the primary care provider should know. JAANP. 2014; 26(1):5-12. https://doi.org/10.1002/23276924.12083

9. Grazina R, Andrade R, Santos FL. Return to play after conservative and surgical treatment in athletes with spondylolysis: A systematic review. Physic Thera Sport. 2019;37: 34-43. https://doi.org/10.1016/j.ptsp.2019.02.005

10. ian Simpson BS. Low Intensity Pulsed Ultrasound.

11. Alyazedi FM, Khan MS, Al MAH. Effect of Ultrasound and Exercise together and TENS alone in the Management of Chronic back pain.

12. Ball JR, Harris CB, Lee J. Lumbar spine injuries in sports: review of the literature and current treatment recommendations. Sports Med Open. 2019; 5(1):1-4. https://doi.org/10.1186/s40798-019-0199-7

13. Russo M, Deckers K, Eldabe S. Muscle control and non-specific chronic low back pain. Neuromod: Tech Neural Interface. 2018; 21(1):1-9. https://doi.org/10.1111/ner.12738

14. Ebadi S, Henschke N, Forogh B. Therapeutic ultrasound for chronic low back pain. $\begin{array}{lllll}\text { Cochrane Database of } & 2020 .\end{array}$ https://doi.org/10.1002/14651858.CD009169.pub3

15. Rubira APFDA, Rubira MC, Rubira LDA. Comparison of the effects of low-level laser and pulsed and continuous ultrasound on pain and physical disability in chronic nonspecific low back pain: a randomized controlled clinical trial. Adv Rheuma. 2019;59:12-16. https://doi.org/10.1186/s42358-019-0099-z 
16. Zhou X-Y, Zhang X-X, Yu G-Y. Effects of low-intensity pulsed ultrasound on knee osteoarthritis: A meta-analysis of randomized clinical trials. BioMed Res Int. 2018; 18; 23-26 https://doi.org/10.1155/2018/7469197

17. Jia L, Wang Y, Chen J, Chen W. Efficacy of focused low-intensity pulsed ultrasound therapy for the management of knee osteoarthritis: a randomized, double blind, placebo-controlled trial. Sci Rep. 2016; 6: 1-9. https://doi.org/10.1038/srep35453

18. Raju PS, Ramu C, Harshavardhan N. Effects of low intensity pulsed ultrasound to reduce the effusion volumes and pain with knee osteoarthritis: a randomized controlled trial. Int J Res Ortho.2019; 5: 77-79.

19. Munajat M, Nordin NAM, Yahya NHM. Effects of low-intensity pulsed ultrasound on recovery of physical impairments, functional performance and quality of life after total knee arthroplasty: Protocol for a quasi-experimental study. Med. 2019;98-101. https://doi.org/10.1097/MD.0000000000017045

20. D'vaz A, Ostor A, Speed C. Pulsed low-intensity ultrasound therapy for chronic lateral epicondylitis: a randomized controlled trial. Rheuma.2006; 45:566-70. https://doi.org/10.1093/rheumatology/kei210

21. Tehranchi A, Badiee M, Younessian F. Effect of Low-intensity pulsed ultrasound on postorthognathic surgery healing process. Annals Maxillo Surg. 2017; 7: 25-28. https://doi.org/10.4103/ams.ams_57_17

22. Warden S, Metcalf B, Kiss Z. Low-intensity pulsed ultrasound for chronic patellar tendinopathy: a randomized, double-blind, placebo-controlled trial. Rheuma. 2008;47:467-71. https://doi.org/10.1093/rheumatology/kem384

Table 1: Baseline characteristics of patients

Variables

Age (years)

Height (cm)

Weight (kg)
LIPUS Group

$30.47 \pm 5.01(23-$

$162.03 \pm 7.86$

$165.20 \pm$

0.20

6.38

$60.88 \pm 7.59$

$66.88 \pm 8.30$

0.30 
Body mass index

$22.53 \pm 2.12$

$23.06 \pm 1.34$

0.39

Gender

Male

$14(82 \%)$

$15(88 \%)$

0.62

Female

$3(18 \%)$

$2(12 \%)$

Data are expressed as the mean \pm standard deviation; LIPUS: Low-intensity pulsed ultrasound; RPT: Routine physical therapy; ${ }^{*} \mathrm{p}<0.05$

Table 2: Within and between group differences for pain and functional disability at $12^{\text {th }}$ week $\& 20^{\text {th }}$ week follow-up from baseline

$\begin{array}{ccc}\text { Outcome } & \begin{array}{c}\text { Assessment } \\ \text { point }\end{array} & \text { LIPUS Group }\end{array}$

Mean SD Mean SD

$\begin{array}{lllll}\text { Baseline } & 7.65 & 0.93 & 6.06 & 1.02\end{array}$

\begin{tabular}{|c|c|c|c|c|c|}
\hline Pain & $12^{\text {th }}$ week & 4.47 & 1.73 & 5.82 & 1.51 \\
\hline & $20^{\text {th }}$ week & 1.47 & 0.80 & 3.82 & 1.2 \\
\hline
\end{tabular}

Within $\quad$ Mean $(95 \% \quad$ P value $\quad$ Mean $(95 \% \quad$ P value

$\begin{array}{lll}\text { group } & \text { CI) } & \text { CI) }\end{array}$

change

$\begin{array}{ccccc}0-12 \text { weeks } & 3.18(2.2-4.2) & <0.001 & 0.24(0.6-1.0) & 0.543 \\ 0-20 \text { weeks } & 6.18(5.5-6.8) & <0.001 & 2.23(1.5-3.0) & <0.001 \\ \begin{array}{c}12-20 \\ \text { weeks }\end{array} & 3.00(2.2-3.8) & <0.001 & 2.00(1.1-2.9) & <0.001 \\ & & & & \end{array}$

$\begin{array}{llllll}\text { Functional } & \text { Baseline } & 60.65 & 10.61 & 40.65 & 13.55\end{array}$

Disability

\begin{tabular}{|c|c|c|c|}
\hline $12^{\text {th }}$ week & 32.41 & 7.61 & 39.65 \\
\hline $20^{\text {th }}$ week & 21.18 & 12.23 & 30.65 \\
\hline $\begin{array}{l}\text { Within } \\
\text { group } \\
\text { change }\end{array}$ & $\begin{array}{c}\text { Mean }(95 \% \\
\text { CI })\end{array}$ & $P$ value & $\begin{array}{c}\text { Mean }(95 \% \\
\text { CI) }\end{array}$ \\
\hline
\end{tabular}

0-12 weeks $28.24(23.7-\quad<0.001 \quad 1.00(2.0-4.0) \quad 0.495$ 


\begin{tabular}{|c|c|c|c|}
\hline 0-20 weeks & $\begin{array}{c}39.47(31.8- \\
47.1)\end{array}$ & $<0.001$ & $\begin{array}{c}10.00(0.3- \\
19.7)\end{array}$ \\
\hline $\begin{array}{l}12-20 \\
\text { weeks }\end{array}$ & $\begin{array}{l}11.24(5.7- \\
16.7)\end{array}$ & $<0.001$ & $\begin{array}{l}9.00(0.0- \\
17.9)\end{array}$ \\
\hline
\end{tabular}

Data are expressed as the mean \pm SD: standard deviation; LIPUS: Low-intensity pulsed ultrasound; RPT: Routine physical therapy; *p<0.05 
